\title{
Ingénieries ou Singeries?
}

\section{Vincent Menuz ${ }^{a}$, \\ Johann Roduit ${ }^{b}$}

a Postdoctorant en bioéthique, Université de Montréal

b Doctorant en droit et éthique biomédicale, Université de Zurich

A ce sujet voir aussi

«Et encore....» à la page 934 .
Des chercheurs en éthique se font du souci pour le réchauffement climatique, à tel point qu'ils ont suggéré en février dernier, dans un article intitulé «Human Engineering and Climate Change» (Ingénierie Humaine et le Changement Climatique), d'utiliser différentes technologies, notamment le génie génétique, afin de rendre les êtres humains respectueux de l'environnement [1]. termes, transformer technologiquement l'être humain pourrait être une des solutions contre le réchauffement climatique.

Ils esquissent quatre possibilités. Premièrement, comme l'élevage de bétail est responsable de $18 \%$ de l'émission de gaz à effet de serre au niveau mondial, les auteurs proposent de rendre les êtres humains intolérants à la viande. Deuxièmement, comme la

\section{Transformer technologiquement l'être humain pourrait être une des solutions contre le réchauffement climatique?}

Selon eux, les solutions actuelles pour réduire l'émission de gaz à effet de serre, telles que le changement des mentalités et des attitudes face à la question environnementale, la régulation du marché (taxes, impôts) et la géo-ingénierie, sont insuffisantes. Ils suggèrent comme solution l'ingénierie humaine qui «implique la modification biomédicale de l'être humain afin de les rendre meilleurs pour réduire le changement climatique». En d'autres taille est proportionnelle à l'empreinte écologique, ils proposent des interventions qui permettraient de rendre les être humains plus petits, de façon à diminuer cette empreinte. Les auteurs suggèrent de choisir les embryons dont les gènes prédisent une petite taille, de modifier les taux d'hormone de croissance chez les enfants, ou encore de réduire le poids des nouveau-nés grâce à des drogues. Troisièmement, s'appuyant sur certaines études qui montrent que les
Correspondance: Johann Roduit Institut d'Ethique Biomédicale Pestalozzistrasse 24 CH-8032 Zurich

Tél. 0446348386

johann.roduit[at]ethik.uzh.ch

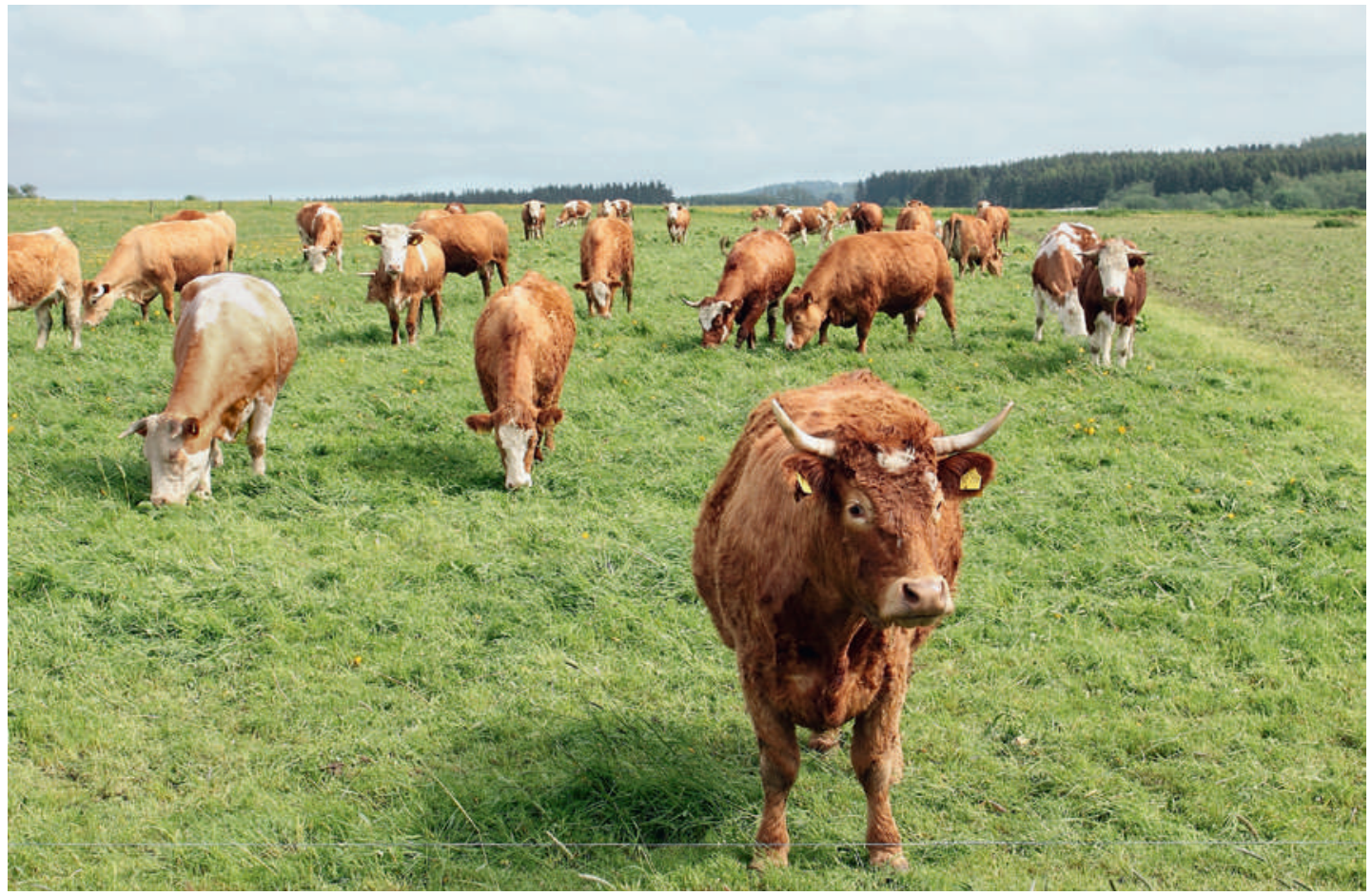

Rendre les êtres humains intolérants à la viande: solution ou provocation? 
femmes «intelligentes» et éduquées font moins d'enfants, ils proposent d'améliorer les capacités cognitives des femmes afin de réduire le nombre d'enfants et ainsi indirectement influencer les changements climatiques. Finalement, ils suggèrent d'améliorer l'empathie et l'altruisme de chacun, par l'intermédiaire de modifications génétiques ou de drogues, afin de rendre l'humain plus sensible aux questions écologiques. liser le DPI, presque nonchalamment en balayant d'un coup de plume les problématiques éthiques liées à l'utilisation de cette technique controversée, on peut légitimement se demander si leur but est moins d'amener une idée novatrice que de provoquer sciemment une réaction de rejet de leurs idées.

Cette étude a toutefois le mérite de mettre en évidence une façon bien singulière de produire de la connaissance: la provocation. Dans cette veine, une

\section{Ce genre de pratique risque de discréditer le travail des chercheurs en bioéthique dans leur ensemble.}

Même si la plupart des propositions de modifications technologiques restent, pour l'instant du domaine de la science fiction, cet article a créé une polémique importante dans le monde anglo-saxon. La position radicale des auteurs a conduit certains commentateurs à les traiter «d'eco-Nazi» et d'eugénistes. Ici, nous soulevons quelques points qui devraient être sérieusement considérés par les auteurs.

Tout d'abord, ils insistent sur le caractère volontaire de ces modifications. Selon eux, aucune de ces interventions ne devrait être imposée. Néanmoins, ils soutiennent que certaines compensations pourraient être envisageables afin de récompenser les individus désirant être modifiés. Ils proposent, notamment, des allègements fiscaux ou des soins de santé parrainés. De telles mesures incitatives sont discutables. En effet, elles peuvent conduire à des problèmes de justice importants. Certaines personnes en situation précaire pourraient envisager d'avoir recours à ces modifications, pour elles-mêmes ou pour leur descendance, dans le but unique de bénéficier de tels avantages. Autrement dit, les plus pauvres seraient plus enclins à être technologiquement modifiés.

Ensuite, les auteurs prennent pour acquis certaines problématiques éthiques qui sont toujours débattues. Ils considèrent par exemple que le diagnostique pré-implantatoire (DPI) «est une pratique acceptée dans beaucoup de cliniques». Or, il se trouve que le DPI est interdit dans un grand nombre de pays. En Suisse, le débat est en cours actuellement et un projet de loi qui autoriserait une telle pratique dans des conditions très particulières est à l'étude. Ainsi, lorsque les auteurs de l'étude proposent d'uti- alternative à celle proposée par les auteurs pourrait être la «désévolution», une proposition du philosophe américain Mark Walker. L'idée serait de retourner à un mode de vie des grands singes des forêts tropicales et serait ainsi une autre façon de participer activement à la lutte contre le réchauffement climatique. Les coûts seraient nuls et aucune technologie particulière ne serait nécessaire. De l'ingénierie à la singerie, il n'y aurait qu'un pas. Plus sérieusement, on peut légitimement se demander si le but de la démarche poursuivie par les auteurs est moins de produire de la connaissance que de se créer une sorte de réputation dans le milieu académique. Il nous semble que ce genre de pratique risque, à terme, de discréditer le travail des chercheurs en bioéthique dans leur ensemble. Si on veut éviter que l'éthique biomédicale figure sur «la liste des professions les moins nécessaires du monde» [2], comme l'a récemment déclaré Andrew Ferguson, il est important de rappeler que le travail académique se construit sur des arguments solides et que la provocation ne constitue généralement pas un argument en soi.

\section{Références}

1 Liao, Sandberg, Roache. Human Engineering and Climate Change. A paraître comme Target Article dans: Ethics, Policy, and Environmnent.

(www.smatthewliao.com/wp-content/ uploads/2012/02/HEandClimateChange.pdf). Consulté le 29 mars 2012

2 Ferguson. Declaring War on Newborns: the disgrace of medical ethics. The Weekly Standard. 17(26). www.weeklystandard.com/articles/declaring-war-newborns_633421.html?nopager=1, consulté le 16 avril 2012. 